\title{
Proceso de Cuidado de Enfermería en una adulta mayor con Síndrome Mieloproliferativo - Policitemia Vera
}

\section{María Martínez-Gil}

RESUMEN: El presente reporte trata de una paciente adulta mayor de 94 años diagnosticada con Policitemia Vera motivo por el cual cuenta con cuidadores asistenciales de forma permanente. El plan de cuidado que se expone está en base a un cuadro de complicación que presentó la paciente. Al realizar la valoración se la encuentra con las funciones vitales alteradas, así como, una alteración a nivel cognitivo. El objetivo fue realizar intervenciones de enfermería tanto a la adulta mayor como al cuidador. Estas intervenciones se realizaron a partir de tres diagnósticos: "Hipertermia", "Confusión Aguda" y "Riesgo de cansancio del rol del cuidador". Se ejecutó el plan en tres meses utilizando el modelo de la valoración de los 13 dominios de la Taxonomía II de NANDA- I y el lenguaje estandarizado de los diagnósticos, intervenciones y resultados de la Taxonomía II. Finalizado el proceso se obtuvo la estabilización de las funciones vitales y la mejoría respecto al nivel cognitivo con el fin de evitar posibles complicaciones. Por otro lado, se logró prevenir y mantener el rol de cuidador mejorando el vínculo cuidador-paciente.

PALABRAS CLAVE: Policitemia Vera; Anciano; Atención de Enfermería.

Citar como: Martínez M. Proceso de Cuidado de Enfermería en una adulta mayor con Síndrome Mieloproliferativo - Policitemia Vera. CASUS. 2017;2(2):127-134.

${ }^{1}$ Hospital Cayetano Heredia. Licenciada en enfermería. Correo electrónico: mbelenmg1605@gmail.com Recibido: 17-04-2017 Aprobado: 27-08-2017 


\section{INTRODUCCIÓN}

La Policitemia Vera (PV) conocida también como enfermedad de Vaquez Osle, fue catalogada en el 2008 por la OMS como una neoplasia mieloproliferativa debido a su origen clonal proveniente de una célula madre pluripotencial de la médula ósea (1). Se caracteriza por el incremento significativo de las células sanguíneas (glóbulos rojos, leucocitos o plaquetas) pero aún no se conoce su causa $(2,3)$.

Estudios realizados a nivel mundial, principalmente en Asia y Europa, indican que la PV suele presentarse entre los 50 y 70 años. Existe una incidencia de 2.8 por cada 100000 varones y un aproximado de 1.3 por cada 100000 mujeres (4). Hasta la fecha en Perú no se tienen datos exactos y actuales del número de casos porque es una patología poco frecuente (2).

Un miembro de la familia que requiere cuidados especiales causa cambios en la familia, ya que en un principio se puede asumir el rol informal de cuidador. Sin embargo ante las crecientes necesidades del paciente se acude a un cuidador formal (enfermero), porque se piensa que puede tolerar o soportar su manejo. Esto puede repercutir de forma negativa en la salud del cuidador una vez superados los recursos disponibles y laborar horas extras (5).

Por ser una patología que suele presentarse en el adulto mayor (4), es de vital importancia actuar cuando los síntomas se manifiestan en una etapa temprana. Con el fin de evitar o disminuir posibles complicaciones tanto a nivel fisiológico (descompensación de las funciones vitales) como a nivel cognitivo. Hay que resaltar que esta enfermedad vuelve dependiente al paciente por lo que el cuidador cumple un rol importante en estos casos.

Frente a la problemática expuesta el presente reporte de caso planteó como objetivo realizar intervenciones de enfermería en el adulto mayor con Síndrome Mieloproliferativo - Policitemia Vera con la finalidad de regularizar posibles daños fisiológicos y cognitivos. Asimismo intervenir con el apoyo del rol cuidador con el fin de brindarle un cuidado humanizado. Para la ejecución se tomó en cuenta el modelo de los 13 dominios de la Taxonomía II de NANDA-I (6), así como las intervenciones planteadas en el Nursing Interventions Classification (NIC) (7) y cumplir los objetivos hallados en el Nursing Outcomes Classification (NOC) (8).

\section{PRESENTACIÓN DEL CASO}

Se presenta el caso de una paciente adulta mayor de 94 años de edad, natural y procedente de Lima con antecedentes de angina, HTA, glaucoma y asma. En un examen auxiliar de sangre se le encontró un recuento de plaquetas de 1015 103/mm3. Razón por la cual se le realizó una biopsia en la médula ósea, entre diversas pruebas donde se le diagnosticó Síndrome mieloproliferativo - Policitemia Vera. La Paciente recibe un tratamiento con anagrelide, hidroxicarbamida y cardioaspirina. Sin embargo, la familia manifestó que ella había iniciado con síntomas bruscos, progresivos y siguió con cefalea intensa, mareos, náuseas y desorientación acompañada de crisis de pánico. Es por ello que se decide contar con un personal de salud domiciliario para el cuidado continuo de la paciente.

\section{VALORACIÓN}

La paciente cuenta con tres cuidadores profesionales de enfermería de forma permanente en su propio hogar debido a su situación actual de salud. La valoración del caso clínico se llevó a cabo clasificando los aspectos positivos y negativos utilizando para ello los 13 dominios de la Taxonomía II de la NANDA-I basándose en el estado actual de dependencia de la paciente y del rol del cuidador (ver table 1).

\section{FASE DIAGNÓSTICA}

Luego de realizada la valoración a la paciente y a sus cuidadores se formularon los diagnósticos de enfermería recopilados de la Taxonomía II de NANDA-I priorizando así tres diagnósticos los cuales permitieron brindar los cuidados de enfermería en respuesta a las necesidades fisiológicas y cognitivas de la paciente y el posible cansancio de roles de los cuidadores.

Reales:

- (00007) Hipertermia r/c enfermedad e/p taquicardia, $\mathrm{T}^{\circ}: 38.5^{\circ} \mathrm{C}$. y piel caliente al tacto. Dominio 11 y Clase 6 .

- (00128) Confusión aguda r/c >60años y enfermedad e/p agitación, desorientación, crisis de pánico. Dominio 5 y Clase 4.

\section{Potencial:}

- (00062) Riesgo de cansancio del rol del cuidador $\mathrm{r} / \mathrm{c}$ actividades de cuidados excesivas. Dominio 7 y Clase 1. 
Tabla 1. Análisis de los Dominios, según aspectos positivos y negativos

\begin{tabular}{|c|c|c|}
\hline Dominios & Positivos & Negativos \\
\hline Promoción de la Salud & Controles regulares de su salud. & $\begin{array}{l}\text { Paciente con policitemia vera. } \\
\text { Antecedentes de asma, HTA, } \\
\text { angina y glaucoma. } \\
\text { T.: } 38.5^{\circ} \mathrm{C} \text { PA.: } 93 / 48 \mathrm{mmHg} \\
\text { FC: } 110 \text { Sat. : } 90 \% \text { FR.: } 25 \text { por } \\
\text { minuto, diarrea aguda. }\end{array}$ \\
\hline Nutrición & $\begin{array}{l}\text { Abdomen b/d no doloroso a la } \\
\text { palpación. }\end{array}$ & $\begin{array}{l}\text { Uso de sonda nasogastrica (SNG) } \\
\text { para alimentación y tratamiento. } \\
\text { Ausencia de piezas dentarias. }\end{array}$ \\
\hline Eliminación e intercambio & Micción espontánea. & $\begin{array}{l}\text { Sialorrea. } \\
\text { Uso de pañal. }\end{array}$ \\
\hline Actividad / Reposo & No se observa. & $\begin{array}{l}\text { Crisis de pánico. } \\
\text { Irritabilidad. } \\
\text { Funciones vitales alteradas } \\
\text { T.: } 38.5^{\circ} \mathrm{C} \text { PA.: } 93 / 48 \text { mmHg FC: } 110 \\
\text { Sat. : } 90 \% \text { FR.: } 25 \text { por minuto. } \\
\text { Uso de sedantes. } \\
\text { Desorientación. } \\
\text { Dependencia total. } \\
\text { Antecedentes de HTA y asma. }\end{array}$ \\
\hline Percepción y cognición & No se observa. & $\begin{array}{l}\text { Deterioro de la audición y del lenguaje } \\
\text { Desorientación } \\
\text { Glasgow 9/15 (Apertura ocular: } 3 \text {, } \\
\text { respuesta verbal: } 2 \text {, respuesta } \\
\text { motora: 4). }\end{array}$ \\
\hline Autopercepción & No se observa. & No se observa. \\
\hline Rol / relaciones & $\begin{array}{l}\text { Familia se encuentra al lado } \\
\text { de la paciente. }\end{array}$ & Actividades de cuidados excesivas. \\
\hline Sexualidad & $\begin{array}{l}\text { Genitales normales según su } \\
\text { edad y sexo. }\end{array}$ & No se observa. \\
\hline $\begin{array}{l}\text { Afrontamiento y tolerancia } \\
\text { al estrés }\end{array}$ & No se observa. & $\begin{array}{l}\text { Irritabilidad. } \\
\text { Crisis de pánico. } \\
\text { Desorientación. } \\
\text { Uso de sedantes. }\end{array}$ \\
\hline
\end{tabular}




\begin{tabular}{l|l|l}
\multicolumn{1}{c|}{ Dominios } & \multicolumn{1}{c}{ Positivos } & \multicolumn{1}{c}{ Negativos } \\
Principios vitales & $\begin{array}{l}\text { Familia refiere pertenecen a la } \\
\text { religión católica. }\end{array}$ & No se observa. \\
\hline Seguridad / protección & No se observa. & $\begin{array}{l}\text { T.: } 38.5^{\circ} \mathrm{C} \mathrm{PA}: 93 / 48 \mathrm{mmHg} \\
\text { FC:110 Sat. : } 90 \% \mathrm{FR} .: 25 \mathrm{x}^{\prime} \\
\text { Piel caliente al tacto. } \\
\text { Vía periférica en brazo derecho } \\
\text { SNG. }\end{array}$ \\
\hline Confort & No se observa. & $\begin{array}{l}\text { Irritabilidad. } \\
\text { Crisis de pánico. } \\
\text { Desorientación. } \\
\text { Uso de sedantes. }\end{array}$ \\
\hline Crecimiento y desarrollo & No se observa. & \begin{tabular}{l} 
No se observa. \\
\hline
\end{tabular} \\
\hline
\end{tabular}

\section{FASE DE PLANIFICACIÓN}

Para la realización del plan de cuidados se tomaron en cuenta los aspectos negativos de la valoración con el fin de identificar los diagnósticos de enfermería. Posteriormente, se seleccionaron las intervenciones de enfermería con la finalidad de regularizar la temperatura corporal y mejorar el estado cognitivo. Por otro lado, se buscó prevenir el cansancio del rol del cuidador (ver tabla 2).

\section{FASE DE EJECUCIÓN}

El plan de cuidados de enfermería se llevó a cabo en tres meses y tuvo como finalidad contribuir con el cuidado del paciente, regularizar la temperatura corporal y mejorar el estado cognitivo. Asimismo, se buscó prevenir el cansancio del rol del cuidador mediante intervenciones para sobrellevar la situación actual de la paciente de tal manera que pueda continuar con sus roles sin sobrecargas físicas y emocionales. Para la elección de las intervenciones en mención se utilizó el lenguaje estandarizado de la Taxonomía NANDA, NIC y NOC. 


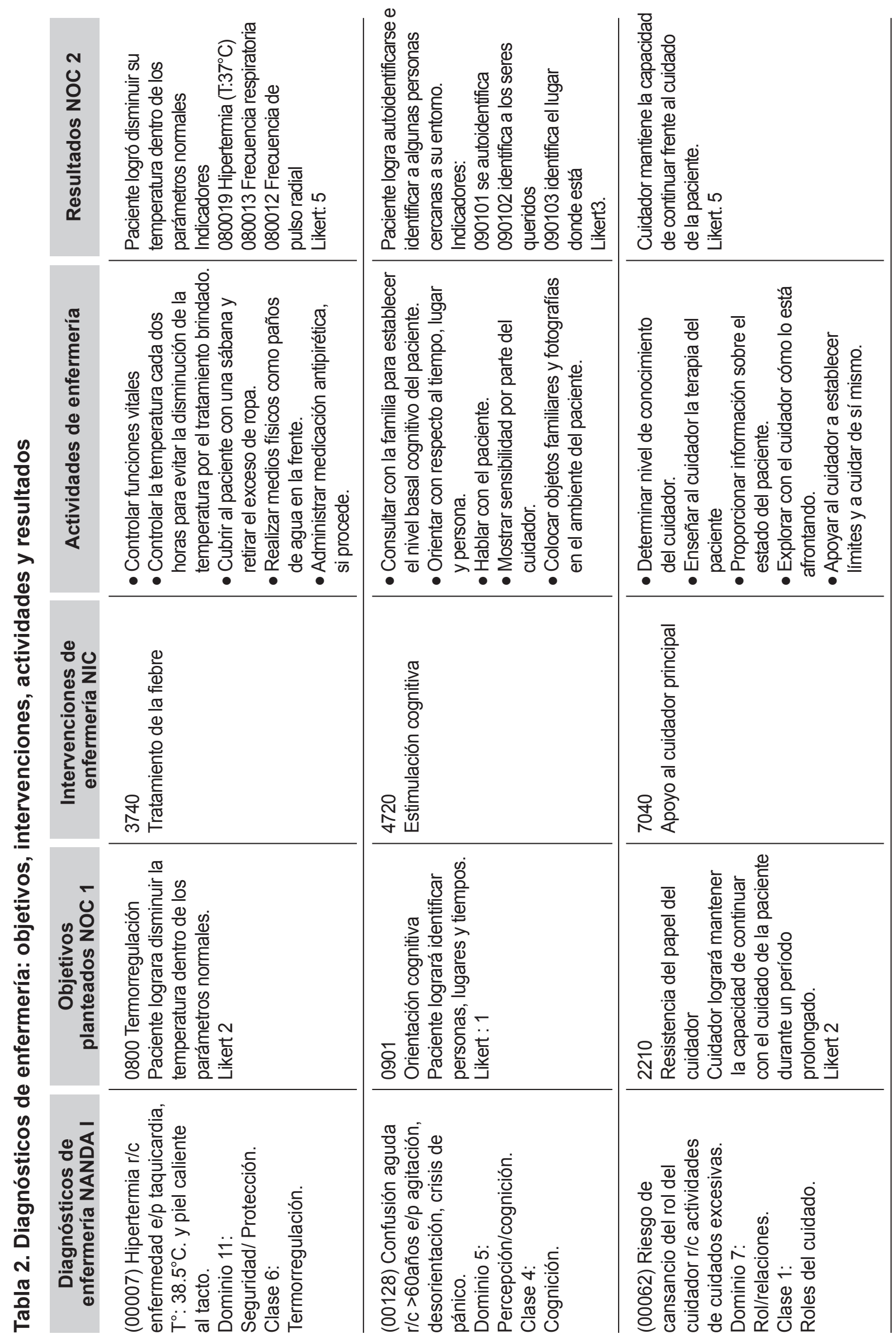




\section{FASE DE EVALUACIÓN}

En la tabla 2 se evidencia la disminución de la temperatura corporal mediante la aplicación de las intervenciones estandarizadas, como el control de signos vitales (temperatura y pulso) y tratar la fiebre a través de medios físicos, uso de medicamentos antipiréticos y el control de funciones vitales. Una vez aplicadas las actividades de enfermería la paciente logra disminuir su temperatura de $38.5^{\circ} \mathrm{C}$ a $37^{\circ} \mathrm{C}$ pasando de un indicador 2 (desviación sustancial de termorregulación) a un indicador 5 (desviación no comprometida de la termorregulación).

En cuanto al diagnóstico de confusión aguda se propuso el objetivo de lograr la orientación cognitiva en lugar, tiempo y espacio. Para ello se realizó la estimulación cognitiva con apoyo de la familia y el resto de los cuidadores para una comunicación continua con la paciente y la adaptación de la unidad de cuidado a través de fotografías y música. Se obtuvo como resultado final un indicador de 3 (moderadamente comprometido) luego de haberla hallado en un indicador 1 (gravemente comprometida).

Respecto al diagnóstico de riesgo de cansancio del rol del cuidador se trabajó mediante actividades que permitan que el cuidador afronte todo tipo de situaciones y mejore los cuidados al paciente. La intervención fue el apoyo al cuidador. Asimismo, se incentivó la actualización sobre los cuidados que debe recibir una paciente con una enfermedad poco común. El indicador inicial mejoró en 3 puntos obteniendo un indicador final de 5 (completamente adecuado).

\section{DISCUSIÓN}

Dada la evaluación del plan de cuidados de enfermería se verifica una mejoría en lo referente a las funciones vitales de la paciente (hipertermia) y función cognitiva; todo ello, con la finalidad de disminuir las posibles complicaciones en el estado de salud del adulto mayor. Adicionalmente, se logró prevenir el cansancio del rol del cuidador a través del apoyo al mismo y de una buena relación paciente-cuidador consiguiendo que el cuidador continúe y colabore en la recuperación y mantenimiento del estado de salud de la paciente sin perjudicarse ambos.
Respecto a los cuidados brindados al paciente para el manejo de la temperatura corporal la literatura reporta que un buen control de la temperatura identifica una fiebre a tiempo y poder intervenir sin aplazar horas de dolor al paciente. Asimismo estas intervenciones se realizaron mediante el uso de medios físicos (paños húmedos, baños, aligerar prendas, entre otros) junto a la administración de antipiréticos que contribuyen a la disminución de la temperatura (9). Por ello, se puede inferir que los medios físicos conjuntamente a la administración de medicamentos, como intervención de enfermería, actúan de manera eficaz en el control de la temperatura (9).

Por otro lado, respecto a la confusión aguda que presentó la paciente se trabajó con la intervención de la estimulación cognitiva y el resultado fue favorable $(10,11)$. Otras investigaciones demostraron efectos beneficiosos producto de la aplicación de esta intervención mediante una visión preventivo-promocional la cual mejoraría la retención de memoria y atención en el adulto mayor $(12,13)$. Esta intervención sería más efectiva antes de un daño a nivel cognitivo. De esta manera el paciente se mantendrá orientado como por ejemplo: reconociendo a sus seres más cercanos, sus cuidadores, etc. (13).

Para el diagnóstico de riesgo de cansancio del rol del cuidador se trabajó mediante actividades que permitieron que el cuidador afronte todo tipo de situaciones respecto a los cuidados de la paciente. La intervención fue el apoyo al cuidador obteniendo así el resultado deseado. Estudios muestran que la familia debe considerar el nivel de sobrecarga que puede tener un cuidador. Incluso el cuidador mismo no debe someterse o soportar estas cargas. Es por ello la importancia de la intervención apoyo al cuidador con el fin de satisfacerlo $(5,14)$. Cabe resaltar que el cuidado es una labor compleja que conlleva, no solo a un desgaste físico, sino también emocional. Es aquí donde el apoyo al profesional de enfermería resulta una intervención primordial, logrando fortalecer el vínculo cuidador-paciente (15). 
Cabe resaltar las fortalezas durante la ejecución del plan de cuidados como son la accesibilidad de la familia para poder trabajar, la disponibilidad de tiempo para el seguimiento del caso, así como el haber podido contar con un ambiente adecuado para la recuperación de la paciente.

Se recomienda realizar estudios donde se evalué la eficacia de los NIC en el proceso de cuidados con el fin de evidenciarla científicamente y contribuir en el desempeño del enfermero. Por otro lado, el profesional de enfermería no solo debe enfocarse en el aspecto fisiopatológico sino en otros aspectos como es el aspecto cognitivo con el fin de mejorar la calidad de vida del paciente evitando el deterioro acelerado de la memoria, ya que el cuidado de un paciente adulto mayor debe ser integral. Por otro lado, el cuidado especial que tienen estos pacientes muchas veces sobrecarga tanto física como emocionalmente al personal de enfermería por lo que se motiva una atención tanto para el cuidador, como del paciente con el fin de mejorar las intervenciones. Ante la evaluación se concluye haber logrado una mejoría respecto a la función cognitiva de la paciente logrando que la misma se autoidentifique e identifique a los familiares más cercanos. Asimismo, se logró estabilizar las funciones fisiológicas (temperatura y pulso) manteniéndolas entre los parámetros normales evitando posibles complicaciones. De igual manera, se logró prevenir el riesgo del cansancio del rol del cuidador, manteniendo y reforzando la relación cuidador-paciente.

\section{REFERENCIAS BIBLIOGRÁFICAS}

1. Tefferi A, Vardiman J. Classification and diagnosis of myeloproliferative neoplasms: The 2008 World Health Organization criteria and point-of-care diagnostic algorithms. Leukemia. 2008;22(1):14-22.

2. Prchal JT, Prchal JF. Polycythemia vera. In: Lichtman MA, Kipps TJ, Seligsohn U, et al. Williams Hematology. McGraw-Hill Medical; 2010.p. 86.

3. Fernández N, Fundora T, Macías I. Policitemia Vera. Experiencias en el diagnóstico y tratamiento en el Instituto de Hematología e Inmunología. Rev Cubana Hematol Inmunol Hemoter. 2011;27(1):77-90.

4. Tefferi A, Spivak JL. Polycythemia Vera: Scientific advances and current practice. Semin Hematol. 2005;42(4):206-20.

5. López $\mathrm{M}$, Orueta R, Gómez $\mathrm{S}$, Sánchez A, Carmona J, Alonso F. El rol de cuidador de personas dependientes y sus repercusiones sobre su calidad de vida y su salud. Rev Clin Med Fam. 2009;2(7), 332-339.
6. Herdman TH, Kamitsuru S. NANDA International . Diagnósticos Enfermeros:Definiciones y Clasificación, 2015-2017. 10a ed. Barcelona: Elsevier; 2015.

7. Bulechek GM, Butcher HK, Dochterman JM, Wagner CM. Clasificación de Intervenciones de Enfermería (NIC). 6ta ed. Barcelona: Elsevier; 2014.

8. Moorhead S, Johnson M, Maas ML, Swanson E. Clasificación de Resultados de Enfermería (NOC). 5ta ed. Barcelona: Elsevier; 2014.

9. Guinart N, López J ¿Qué sabemos de la fiebre. Rev Cubana Med Gen Integr. 1997;13(2):159-165.

10. Alves J, Batista D, Paúl C, Alves M, Macedo M. Efectos de la estimulación cognitiva sobre las personas mayores en el ámbito comunitario. Enfermería Clínica. 2016;26(2):111-120.

11. Rey A, Canales I, Táboas M. Calidad de vida percibida por las personas mayores. Consecuencias de un programa de estimulación cognitiva a través de la motricidad «Memoria en movimiento. Rev Esp Geriatr Gerontol. 2011;46(2):74-80.
12. Bohórquez V, Castañeda A, Trigos L, Orozco L, Camargo F. Eficacia de la intervención enfermera para cuidadores con cansancio del rol del cuidador. Rev Cubana Enfermer. 2011;27(3):210-219.

13. Cáceres FM, Bohórquez F, Puerto HM, Smith D. Eficacia de las intervenciones de enfermería para disminuir el temor en personas que inician tratamiento para el cáncer. Un ensayo clínico controlado. MedUNAB. 2004;7:161(5). 


\section{Nursing Care Process in an older adult with Myeloproliferative Syndrome - Polycythemia Vera}

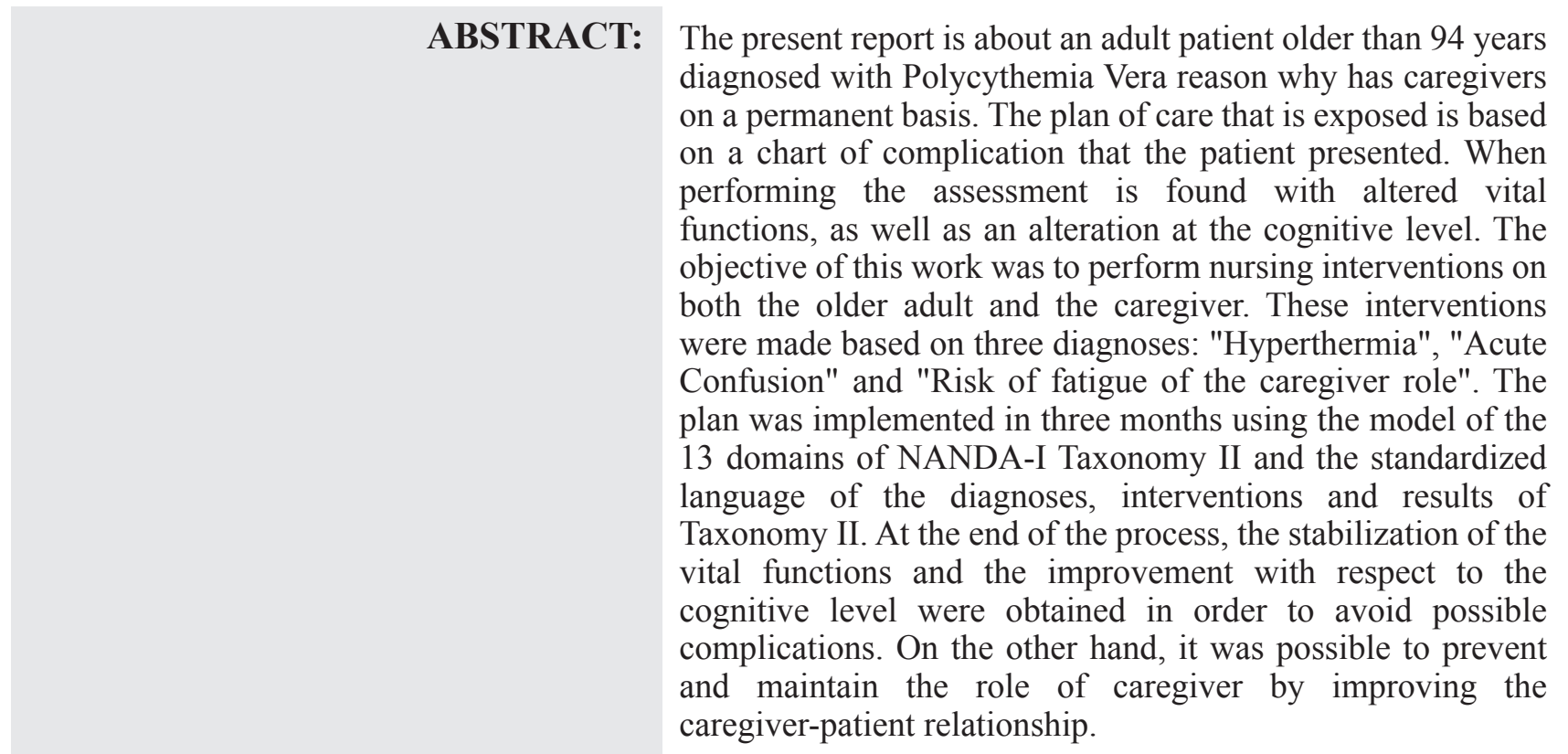

KEY WORDS: Polycythemia Vera; Aged; Nursing Care 